\title{
Reacciones adversas a L-asparaginasa en pacientes con leucemia linfoblástica aguda
}

\author{
Mónica Kyonen L ${ }^{1 a}$, Isabel Folatre $B^{2,3}$, Paolina Zolezzi $\mathbf{R}^{3}$, \\ Viviana Badilla $\mathrm{M}^{2 \mathrm{~b}}$, Francisco Marín $\mathrm{H}^{4 c}$. \\ Adverse reactions to $L$-asparaginase in \\ children with acute lymphatic leukemia
}

Background: The low notification of Adverse Drug Reactions (ADR) underscores the need for pharmacological surveillance systems that allow their detection, evaluation, prevention and registry especially in patients receiving multiple medications. Aim: To communicate the ADR observed with the use E coli asparaginase (E coli ASP) in children with Acute Lymphatic Leukemia (ALL). Patients and Methods: Cross-sectional study of clinical records of all the patients aged less than 15 years with $\mathrm{ALL}(\mathrm{n}=52)$ and treated between January 1996 and April 2000. The suspicion of ADR to E coli ASP was evaluated using the Karch and Lasagna algorithm. Probable and definitive cases of ADR to E coli ASP were subjected to a protocol of clinical and epidemiological data. The risk factors associated to ADR to E coli ASP, were also determined. Results: Fifty children aged 1 to 13 years (33 men) were studied. In 20 (40\%), an ADR to E coli ASP, was suspected. After the use of the algorithm, the ADR was considered definite in two patients, probable in 17 and possible in one. The registered clinical events were urticaria in 17, anaphylactic shock in four, transient alterations of hepatic functions test in seven and hypofibrinogenemia in four. The test of cutaneous sensitivity to the administration of E coli ASP, performed prior to the administration of the drug, were positive in nine of the 20 children with adverse reactions. No children died. Conclusions: The application of the Karch anbd Lasagna algorithm, allowed us to objectively classify suspected cases of ADR by E coli ASP (Rev Méd Chile 2006; 134: 1530-4).

(Key words: Asparaginase; Drug toxicity; Leukemia, lymphocytic)

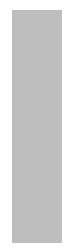

\begin{abstract}
aQuímico Farmacéutico
bEnfermera Universitaria

cBioestadístico
\end{abstract}

Recibido el 12 de julio, 2005. Aceptado el 18 de mayo, 2006.

${ }^{1}$ Unidad de Farmacia y ${ }^{2}$ Servicio de Pediatría, Hospital Clínico Regional Valdivia. ${ }^{3}$ Instituto de Hematología «R. Virchow», Universidad Austral de Chile. ${ }^{4}$ Instituto de Salud Pública, Facultad de Medicina, Universidad Austral de Chile.
$\mathrm{E}^{\mathrm{s}}$ el año 1969, la Organización Mundial de la Salud (OMS) definió reacción adversa a medicamento (RAM) como «cualquier efecto dañino, no deseado, ejercido por un fármaco administrado a

Correspondencia a: QF Mónica Kyonen L Unidad de Farmacia, Hospital Clínico Regional Valdivia. Simpson \# 850, Valdivia, Chile. Fono: 63-293000. E mail: mkyonen@yahoo.com humanos en dosis usadas con fines profilácticos, diagnósticos y terapéuticos o para la modificación de una función fisiológica». La probabilidad de identificar RAM con una frecuencia de 1:1.000 es remota ${ }^{1,2}$, esperándose un aumento significativo en relación con el lanzamiento de nuevos fármacos, especialmente en pacientes que requieren de terapias múltiples, intensivas $\mathrm{y}$, en ocasiones, 
prolongadas. Esta situación obliga a contar con un sistema de registro de RAM que permita recolectar y estudiar la información obtenida, con el objeto de asegurar el correcto uso de los medicamentos, desarrollándose así la farmacovigilancia, cuyo objetivo es contribuir a la detección, evaluación y prevención de las RAM en humanos ${ }^{3}$. En el Hospital Clínico Regional de Valdivia (HCRV), se registran espontáneamente las RAM en el formulario amarillo de notificación de sospecha de reacciones adversas a medicamentos, elaborado por el Centro Nacional de Información de Medicamentos y Farmacovigilancia (CENIMEF) del Instituto de Salud Pública (ISP), dependiente del Ministerio de Salud (MINSAL/CHILE). Los datos así recopilados son enviados, desde los servicios clínicos a la Unidad de Farmacia del HCRV y al CENIMEF, con el fin de notificar y evaluar las RAM a nivel regional y nacional, siendo luego esta información referida al Centro Colaborador de Monitorización Internacional de RAM de la OMS en Uppsala, Suecia $^{4,5}$. El HCRV es centro de referencia nacional para el tratamiento del cáncer infantil de las regiones X, XI y XII del país. Los menores de 15 años con diagnóstico de leucemia linfoblástica aguda ingresan al Estudio y Tratamiento de la Leucemia Linfoblástica Infantil/Programa Infantil Nacional de Drogas Antineoplásicas (LA/PINDA/ 96/MINSAL) ${ }^{6}$. Uno de los medicamentos incluidos en este tratamiento es L-asparaginasa (ASP), enzima obtenida de Escherichia coli (tipo EC-2), de efectos adversos ampliamente conocidos ${ }^{7-10}$. Es de nuestro interés comunicar en menores de 15 años, con LA tratados, las RAM observadas al administrar E coli ASP.

\section{MateRIAL y MÉTOdo}

Estudio transversal de las fichas clínicas de todos los menores de 15 años con diagnóstico LA (n =52), tratados en el HCRV, entre el 10 de enero de 1996 y el 30 de abril de 2000, bajo el protocolo de LA/PINDA/96/MINSAL ${ }^{6}$. El Protocolo I de Inducción/Fase I establece el uso de E coli ASP en dosis de $5.000 \mathrm{UI} / \mathrm{m}^{2} /$ día/iv, administrado en una hora $\mathrm{x}$ $8 \mathrm{c} / 72 \mathrm{~h}$ y $10.000 \mathrm{UI} / \mathrm{m}^{2} /$ día/iv x $4 \mathrm{c} / 72 \mathrm{~h}$ en el Protocolo II de intensificación de la Inducción/Fase I, previa prueba de sensibilidad cutánea. Todos los menores ( $\mathrm{n}=52$ ) reciben E coli ASP en un esquema que, además, incluye prednisona po, metotrexato it, vincristina iv, daunorrubicina iv y, en el protocolo II, dexametasona po, vincristina iv y doxorrubicina iv. Los antecedentes epidemiológicos, clínicos, de laboratorio y tratamiento extraídos se transcribieron a una ficha de recolección de datos de estudio, elaborada para tales efectos. Se consideró RAM por E coli ASP a la presencia de prueba cutánea positiva, urticaria, falla respiratoria aguda, shock anafiláctico, alteraciones de pruebas de función hepática y de coagulación. Los datos obtenidos que permitieron sospechar RAM por E coli ASP, fueron sometidos al algoritmo Karch y Lasagna ${ }^{11,12}$. La aplicación del algoritmo permite evaluar la relación de causalidad ante una sospecha de RAM, ya que establece una serie de preguntas cerradas que generalmente deben responderse «í» 0 no». Las respuestas a las preguntas realizadas por los dos investigadores principales fueron clasificadas según categoría de probabilidad en definitiva, probable, posible, condicional, o no RAM, bajo criterios establecidos en tabla de decisión Karch y Lasagna $^{12}$. RAM definitiva: evento clínico que reaparece tras la reexposición al medicamento; RAM probable: presentado el evento clínico no hay reexposición al medicamento; RAM posible: evento clínico que puede ser explicado por otra causa, ya sea enfermedad u otro medicamento o la información entregada sobre suspensión del fármaco no es suficientemente clara; RAM condicional: evento clínico que se explica por otra causa. Se utilizaron antihistamínicos y corticoides iv en las RAM. En los pacientes RAM probable se suspendió E coli ASP, reemplazándose por Erwinia L-asparaginasa. Los factores de riesgo asociados a RAM por E coli ASP se analizaron mediante odds ratio utilizando el programa EPI INFO versión 6.04 .

\section{RESULTADOS}

Se evaluaron los datos registrados en 52 menores, excluyéndose 2 por traslado a otros centros $n=50$. Edad: 1 a 13 años, 33 varones. La ficha de recolección de datos RAM/ASP fue sometida al programa estadístico, resultando un grupo de pacientes con RAM 20/50 y otro grupo sin RAM de 30/50. Las sospechas de RAM por E coli ASP en $20 / 50$, con una incidencia de $40 \%$, fueron evaluadas por los dos investigadores, aplicando el 
algoritmo Karch y Lasagna, valorando cada pregunta de éste. Resultados según categoría de probabilidad: RAM definitiva $=2$; RAM probable =17; RAM posible =1. En Tabla 1, se consignan variables clínicas de pacientes con y sin RAM por E coli ASP. Algunos pacientes cursaron con más de una manifestación clínica o de laboratorio. Todos los pacientes con RAM probable ${ }^{17}$ presentaron urticaria de rápida aparición después de haber recibido entre 1-6 dosis (8/17) y 7-12 dosis (9/17) de E coli ASP, agregándose shock anafiláctico en 2/17. En todos se suspendió E coli ASP, reemplazándose en 9 por Erwinia L-asparaginasa. Recibieron antihistamínicos más corticoides iv 12/17. Los dos casos de RAM definitiva presentaron shock anafiláctico al iniciar la administración de la 8a dosis de E coli ASP, con substitución por Erwinia L-asparaginasa, recibiendo tratamiento similar. No se presentaron reacciones adversas frente al uso de Erwinasa L-asparaginasa. No se observó mortalidad.

Las pruebas de sensibilidad cutánea previas a la administración de E coli ASP se interpretaron como positivas en 9/20. Al presentar RAM 13/20 estaban recibiendo vincristina y E coli ASP y 8/20 prednisona y E coli ASP. Los factores de riesgo asociados a RAM por E coli ASP expresados mediante la medida de asociación odds ratio se encuentran en Tabla 2. Las tasas por año de RAM por ASP se expresan en Tabla 3.

Tabla 1. Variables clínicas de pacientes con presencia o no de reacciones adversas a asparaginasa

\begin{tabular}{|c|c|c|c|c|}
\hline Variables & & $\mathrm{N}^{0} \mathrm{RAM}$ & $\mathrm{N}^{0}$ No-RAM & $\mathrm{N}^{0}$ Total \\
\hline \multirow[t]{2}{*}{ Urticaria } & No & 3 & 21 & 24 \\
\hline & $\mathrm{Si}$ & 17 & 3 & 20 \\
\hline \multirow[t]{2}{*}{ Shock anafiláctico } & No & 16 & 23 & 39 \\
\hline & $\mathrm{Si}$ & 4 & 0 & 4 \\
\hline \multirow[t]{2}{*}{ Prueba L-asp } & No & 11 & 32 & 43 \\
\hline & $\mathrm{Si}$ & 9 & 0 & 9 \\
\hline \multirow[t]{2}{*}{ Dosis RAM } & 1-6 dosis & 8 & 4 & 12 \\
\hline & $7-12$ & 12 & 28 & 40 \\
\hline \multirow[t]{2}{*}{ Asociación vincristina } & No & 7 & 3 & 10 \\
\hline & $\mathrm{Si}$ & 13 & 28 & 41 \\
\hline \multirow[t]{2}{*}{ Asociación prednisona } & No & 12 & 1 & 13 \\
\hline & $\mathrm{Si}$ & 8 & 31 & 39 \\
\hline \multirow[t]{2}{*}{ Asociación metotrexato } & No & 16 & 0 & 16 \\
\hline & $\mathrm{Si}$ & 4 & 32 & 36 \\
\hline
\end{tabular}

Tabla 2. Factores de riesgo asociados a la presencia de reacciones adversas medicamentosas a asparaginasa

\begin{tabular}{|lcc|}
\hline RAM & Odds Ratio $(\mathrm{OR})$ & IC $95 \%$ \\
\hline Urticaria & 39,67 & $(5,7-364,6)$ \\
Shock anafiláctico & NO DEF* & NO DEF* \\
Prueba ASP & NO DEF* & NO DEF* \\
No dosis de L-ASP & 4,67 & $(0,99-23,38)$ \\
Asociación a prednisona & 46,50 & $(4,82-1111,29)$ \\
Asociación a vincristina & 5,03 & $(0,94-29,9)$ \\
Asociación a metotrexato & NO DEF* & NO DEF* \\
\hline
\end{tabular}

*No se logró definir OR. 
Tabla 3. Tasa de reacciones adversas por año

\begin{tabular}{|lccc|}
\hline Año & № de RAM/año & № de casos total/año & Tasa (\%) \\
\hline 1996 & 3 & 6 & 50 \\
1997 & 6 & 11 & 54 \\
1998 & 5 & 12 & 42 \\
1999 & 3 & 8 & 38 \\
2000 & 3 & 13 & 23 \\
\hline
\end{tabular}

\section{DisCUSIÓN}

El punto crítico en RAM es establecer la relación causal entre el fármaco sospechoso y el evento clínico observado, ya que con frecuencia las reacciones son de carácter ambiguo, generalmente el paciente está recibiendo más de una droga y no hay pruebas específicas para determinar relación causaefecto. La aplicación del algoritmo Karch y Lasag$\mathrm{na}^{12}$, permitió clasificar las sospechas de RAM por $\mathrm{E}$ coli ASP 40\% (20/50), reportándose una incidencia variable de $5 \%$ a $33 \%{ }^{13-16}$ con predominio de RAM probables. Entre las RAM por ASP conocidas destacan las reacciones alérgicas con $29,5 \% 13,16,17$, considerándose que corresponden a una toxicidad mediada inmunológicamente, que involucraría la producción de inmunoglobulinas $\mathrm{G}$ y $\mathrm{M}$, con una expresión clínica que va desde urticaria a shock anafiláctico de ocasional curso fatal. Frente a la administración de E coli ASP, el signo de alerta de RAM estuvo dado predominantemente por la aparición de urticaria [odds ratio 39,67 IC 95\% (5,7364,6)]. El shock anafiláctico (4/20), expresión máxima de RAM, se presentó entre 15 y $60 \mathrm{~min}$ después de la administración de E coli ASP y pese al compromiso respiratorio severo y agudo los cuatro pacientes, no necesitaron intubación ni ventilación mecánica, evolucionando con buena respuesta clínica al tratamiento iv con corticoides y antihistamínicos, agregándose adrenalina iv en uno de los casos. Los dos casos clasificados como RAM definitiva, con antecedentes uno de urticaria y el otro con prueba de sensibilidad cutánea positiva, al ser expuestos a la 8a dosis de E coli ASP presentaron RAM más severa. Se han descrito alteraciones neurológicas en $4,4 \%^{18}$ y en 2,9\% alteración de la función hepática, encontrando nosotros 38,9\% (7/ 18) de aumento de las transaminasas, principalmente GPT, pero de rápida normalización. ASP como fármaco único disminuye en forma significativa la concentración plasmática de casi todas las proteínas e inhibidores de la coagulación, con una reducción significativamente mayor de los niveles de antitrombina III ${ }^{19}$. Se ha observado un aumento de la generación de trombina durante las primeras semanas de tratamiento con E coli ASP, por lo que el descenso de antitrombina III podría reflejar la disminución de la síntesis proteica y un mayor consumo $^{19}$. Se ha descrito $2,2 \%$ de hipofibrinogenemia ${ }^{15,16}$, encontrando en nuestro estudio $30,7 \%$ (4/ 13), con tiempo de protrombina (TP), tiempo de tromboplastina (TTPA) y producto de degradación del fibrinógeno dímero $\mathrm{D}$ (PDF) prolongado 0 disminuido, hecho que hace necesario medir niveles de antitrombina III pre y postratamiento con E coli ASP. Para seleccionar a los pacientes alérgicos a E coli ASP, se recomienda realizar una prueba previa de sensibilidad cutánea ${ }^{6}$, negativa en 11/20. Estos resultados nos llevaron a modificar nuestra conducta, suspendiendo la prueba, administrando desde el año 1999, E coli ASP bajo estricta observación clínica y laboratorio con premedicación ev de antihistamínicos y corticoides en casos con antecedentes de sensibilización previa. $\mathrm{Al}$ analizar las tasas RAM/ASP/año, éstas expresan una disminución progresiva del $\mathrm{n}$ \%/casos de RAM/ASP respecto al total de pacientes expuestos al fármaco, a medida que transcurren los años del estudio, atribuible a las medidas tomadas. No observamos reacciones adversas en 11 pacientes que recibieron Erwinasa Lasparaginasa, pese a RAM descritas similares, completando los pacientes las dosis restantes de ASP según protocolo. No se contó con disponibilidad de Erwinasa en todos los casos. Uno de los principales factores de riesgo asociado y en relación a la presencia de RAM estaría dado por el número de dosis de exposición a ASP [odds ratio 4,67 IC $95 \%(0,99-23,38)]$, con un promedio de 11,5 dosis 
de E coli ASP administradas ${ }^{15}$. El rango establecido en nuestros pacientes de 7 a 12 dosis recibidas es similar a lo reportado ${ }^{15}$. Otro de los factores de riesgo asociado a la presencia de RAM estuvo dado por la administración de prednisona [odds ratio 46,5 IC 95\% (4,82-1111,29)]. En los protocolos actuales se distancia la administración de vincristina y ASP al menos por $24 \mathrm{~h}$ para disminuir la urticaria, obteniendo nosotros un odds ratio 5,03. La aplicación del algoritmo Karch y Lasagna, nos ha llevado a clasificar objetivamente las sospechas de RAM por

\section{REFERENCIAS}

1. Drug Interactions and Adverse Drug Reactions. En: Sammons J., 6 ${ }^{\underline{a}}$ Ed. Drug Evaluations. USA: American Medical Association 1986; 29-51.

2. GRIFFIn J, WeBER J. Voluntary Systems of Adverse Reaction Reporting-Part I. Adv Drug React Ac Pois Rev 1985; 4: 213-30.

3. Meyboom R, Lndquist M, Egberts C. An ABC of Drug Related Problems. En: Edwards R et al. Drug safety. Pharmacovigilance in Focus. $2^{\mathrm{a}}$ Ed AIDS International Ltda, 2001: 415-23.

4. Organización Mundial de la Salud. El Monitoreo de la Seguridad de los Productos Medicinales. Guía para el establecimiento y Funcionamiento de Centros de Farmacovigilancia. Buenos Aires, 1997.

5. Capeiá D, Laporte J. La Notificación Espontánea de Reacciones Adversas a Medicamentos. En: Laporte J, Tognoni G. Principios de Epidemiología del Medicamento. 2 ${ }^{\mathrm{a}}$ Ed Barcelona: Masson-Salvat, 1993; 147-50.

6. CAMPBell M. Protocolo de Estudio y Tratamiento de Leucemia Linfoblástica Infantil PINDA. Ministerio de Salud. Chile. 1996.

7. Miscellaneous Antineoplastics. En: Sewester S, Bernie R et al. 47 ed. Drug Facts and Comparisons. St Louis. A Wolters Kluwer Company, 2000; 1920.

8. Antineoplastics and Immunosuppressants. En: Reynolds JEF, Martindale, 32 Ed. The Complete Drug Reference. London Pharmaceutical Press, 1999; 504.

9. Antineoplastic Agents. En: Mc Evoy GK. AHFS Drug Information. Bethesda, MD, American Society of Health-System Pharmacists, Inc, 1998; 739.

10. Antineoplastic Agents. En: Mc Evoy GK. AHFS Drug Information. Bethesda, MD, American Society of Health-System Pharmacists, Inc, 2002; 901.

11. Laporte J, Capelí D. Mecanismo de producción y diagnóstico clínico de los efectos indeseables pro-
ASP, permitiéndonos evaluar la continuidad del protocolo indicado en RAM probables. La implementación de Programas de Farmacovigilancia/ CENIMEF-ISP/HCRV, nos ha obligado a realizar una observación cuidadosa, a pesquisar y notificar las RAM secundarias al uso de quimioterápicos en el tratamiento del cáncer. Nuestra experiencia ha sido un modelo para continuar aplicando estos programas en otros fármacos y otros pacientes, lo que ha dado como resultado una mejoría de la calidad de la atención al paciente.

ducidos por medicamentos. En: Laporte J, Tognoni G, 2aㅡ Ed. Principios de Epidemiología del Medicamento. Barcelona. Masson-Salvat, 1993; 95-106.

12. Karch F, Lasagna L Toward the Operational Identification of Adverse Drug Reaction. En Clin Pharmacol and Ther 1997; 21: 247-54.

13. Barron A, Luke K, HSU E, Torsher L, Deveber L, CAIRNEY A. Toxicity Study of Erwinia Asparaginase as First Line Therapy for Chilhood Acute Lymphocytic Leukemia. Hannnover. Proceedings of the $5^{\text {th }}$ Pharmacology Workshop Satellite to XXIV SIOP Meeting. 1992; 100-4.

14. Drug Evaluation Monograph. Asparaginase. En: Drugdex Information System Denver (CO) staff, Micromedex ${ }^{\circledR}$ Healthcare Series 2003; Vol 122.

15. Drug Evaluation Monograph. Asparaginase. En: Drugdex Information System Denver (CO) staff, Micromedex ${ }^{\circledR}$ Healthcare Series 2005; Vol 123.

16. Foьв P. Citostatics and Immunosuppressive Drugs. En: Dukes M.N.G, Aronson J., 14ª Ed. Meyler's Side Effects of Drugs. Annual 23. Amsterdam: Elsevier, 2000; 1543, 1555, 1559, 1563, 1572.

17. FERRÁNDIZ FoARASTER C. Dermatología Clínica. Mosby-Doyma 1996; 136-47.

18. Eden O, Shaw M, Lumeyman J, Richards S. NonRandomized Study Comparing Toxicity of Escherichia coli and Erwinia Asparaginase in Children with Leukemia. Med Pediatr Oncol 1990; 18: 497-502.

19. Andrew M, Brooker L, Mitchell L. Acquired Antitrombina III Deficiency Secondary to Asparaginase Therapy in Childhood Acute Lymphoblastic Leukemia. Blood Coagulation and Fibrinolysis 1994; 15; Suppl 1: S24-S36.

20. Cancer Drug Manual. BC Cancer Agency Sept 2001; disponible en:http:// www.bccancer.bc.ca/ $\mathrm{cdm} /$ monographs/asparaginase.shtml (consultado el 7 de enero de 2001). 